\title{
Processing the diffusion-weighted magnetic resonance imaging of the PING dataset
}

\author{
Noor B Al-Sharif ${ }^{\mathrm{a}, *}$, Etienne St-Onge ${ }^{\mathrm{b}, *}$, Guillaume Theaud ${ }^{\mathrm{b}}$, Alan C Evans ${ }^{\mathrm{a}}$, \\ Maxime Descoteaux ${ }^{\mathrm{b}}$ \\ ${ }^{a}$ McGill Centre for Integrative Neuroscience (MCIN), McGill University, Canada \\ ${ }^{b}$ Sherbrooke Connectivity Imaging Laboratory (SCIL), Université de Sherbrooke, Canada
}

\begin{abstract}
Diffusion-weighted magnetic resonance imaging (dMRI) allows for the in-vivo assessment of anatomical white matter in the brain, thus allowing the depiction of structural connectivity. Using structural processing techniques and related methods, a growing body of literature has illustrated that connectomics is a crucial aspect to assessing the brain in health and disease. The Pediatric Imaging Neurocognition and Genetics (PING) dataset was collected and released openly to contribute to the assessment of typical brain development in a pediatric sample. This current work details the processing of diffusion-weighted images from the PING dataset, including rigorous quality assessment and fine-tuning of parameters at every step, to increase the accessibility of these data for connectomic analysis. This processing provides state-of-the-art diffusion measures, both classical diffusion tensor imaging (DTI) and more advanced HARDI-based metrics, enabling the evaluation not only of structural white matter but also of integrated multimodal analyses, i.e. combining structural information from dMRI with functional or gray matter analyses.
\end{abstract}

Keywords: diffusion MRI, connectome, tractography, white matter, pediatric development

\section{Introduction}

Brain structure \& development

Post-natal neural development is characterized by widespread structural changes of the brain, in both gray and white matter regions [Sowell et al., 5 2004; Lenroot and Giedd, 2006; Giedd and Rapoport, 2010]. Microstructural changes in brain architecture, such as cortical thickness or myelination, lead to global morphometric changes detectable by magnetic resonance imaging (MRI) techniques, including anatomical images (such as $\mathrm{T} 1$ or $\mathrm{T}^{\star}{ }^{\star}$ weighted) and diffusion-weighted imaging (dMRI). Just as these techniques can be used to

*These authors contributed equally: shared first and corresponding authors 
assess differences between health and disease states, they also provide insight into underlying microstructural changes in the brain that characterize typical development in vivo [Lim and Helpern, 2002; Prayer and Prayer, 2003; Qiu et al., 2015]. Implementing multimodal imaging methods in the assessment of structural development throughout the lifespan enables a comprehensive per-

15 spective of anatomical growth and variance across the whole brain. Research has shown that a major component of post-natal brain development occurs in the strengthening or refining of local and whole-brain white matter connections [Lebel et al., 2008; Jernigan et al., 2011]. Many of these changes are experiencedependent and causally linked with myelination, synaptogenesis and synaptic

20 pruning in different areas of the brain at different developmental periods [Shatz, 1990; Benes et al., 1994; Paus et al., 1999; Stiles and Jernigan, 2010; Deoni et al., 2011]. Such changes in structural white matter provide a compelling case for the use of dMRI in assessing brain development.

\section{Diffusion MRI $\&$ white matter processing}

25 DMRI is a particular MRI sequence that quantifies water displacement in multiple directions. From diffusion-weighted images, local diffusion models are reconstructed to estimate the underlying characteristics of white matter anatomy [Jones, 2008; Descoteaux, 2015]. The dynamic changes of this microstructure in development can be characterized both in structural and func-

so tional analyses [Klingberg et al., 1999; Mukherjee et al., 2002; Olesen et al., 2003; Sotiropoulos and Zalesky, 2019]. These changes, whether localized or not, underlie global changes in whole-brain networks, which form the basis of brain function and behaviour [Nagy et al., 2004]. As such, it is important to evaluate these changes in the context of brain connectivity, using techniques such

35 as dMRI to depict structural white matter changes [Jeurissen et al., 2019]. As of yet, we have a limited understanding of how white matter develops during childhood and adolescence and therefore require more insight into how these structures change over time.

Though the implementation of dMRI techniques has improved our under-

40 standing of anatomical white matter structure, processing of such data is known to be rife with complex challenges [Jones and Cercignani, 2010]. These challenges consist of low spatial resolution, noisy acquisition, MRI gradient-related artifacts and fiber organisation ambiguity (crossing, kissing, fanning) [Jbabdi et al., 2015; Dell'Acqua and Tournier, 2019; Jeurissen et al., 2019]. As such,

45 processing dMRI data needs to be reliable and reproducible to improve dMRI quality and better depict local and global structure of the human brain [Theaud et al., 2020].

\section{PING}

In order to depict stereotypical changes or patterns in structural developso ment, the use of large-scale population-representative datasets is essential. One such dataset is the Pediatric Imaging Neurocognition and Genetics (PING) study: a large-scale developmental cohort of subjects aged between 3 and 22 years, including imaging, cognitive and genetic data. This comprehensive study 
provides a rich multimodal imaging perspective of development, from childhood to early adulthood, and enables the integration of behavioural and genetic information. PING publicly provides both the raw imaging and demographic data, as well as some processed derivatives of these data including cortical thickness, surface area, volumes and some diffusion-tensor measures. However, despite this notable amount of data made available by PING, structural white matter connectivity measures were not included, to our knowledge.

To best take advantage of these large-scale datasets, such data must be made accessible for use beyond their originating research groups. These types of open science practices promote scientific collaboration and discovery, such as the Human Connectome Project (HCP) [Glasser et al., 2013]. The creation and

${ }_{65}$ analysis of large-scale population datasets are arduous tasks best accomplished as collaborative efforts. Sharing data encourages better, more transparent research practices and also minimizes the necessity for repetition in laborious data collection and processing. Therefore, the derivatives of this current processing will be made publicly available and accessible through the National Database

70 for Autism Research (NDAR) repository, a database of the National Institute of Mental Health Data Archive (NDA), which also hosts the original PING dataset.

\section{Methods}

In this section, we specify how the initial PING release and dMRI acquisition were harmonized and formatted [Jernigan et al., 2016]. The pre-processing and 75 processing applied to dMRIs is detailed, along with any processing applied in parallel to the T1-weighted anatomical images. Finally, tractography and connectivity matrix generation are described. As detailed in Jernigan et al. [2016], written consent was obtained from parental guardians for participants below 18 years of age and from the participants themselves otherwise, in accordance with

so IRB protocols. For reproduction purposes, details are listed in the Usage Notes and Code availability sections. Figure 1 illustrates a comprehensive overview of the processing pipeline.

\subsection{Input data collection and formatting \\ Imaging data collection}

85 Original PING data were collected in Digital Imaging and Communications in Medicine (DICOM) format across nine sites on four 3T MR machine types: Siemens TrioTim, GE Discovery, GE Signa, and Philips Achieva. Scanning protocols were harmonized across all scanners and sites to minimize variance across scans. From PING, we gained access to a total of 954 subjects with

90 dMRI scans, 634 of which also have at least 2 or more repeated scans within the same session. In total, 1669 unique acquisitions were used as input for the subsequent processing. 


\section{Anatomical images}

Though the PING dataset contains both $\mathrm{T} 1 \mathrm{w}$ and $\mathrm{T} 2{ }^{\star} \mathrm{w}$ images, only $\mathrm{T} 1 \mathrm{w}$ images are required for the processing described here. $\mathrm{T} 1 \mathrm{w}$ images were collected using a real-time prospective motion correction (3D PROMO) technique to minimize the effects of motion, which is typically widespread in pediatric samples. Subjects were processed only if they had both dMRI and T1w images which passed quality assurance, i.e. minimal motion, no major artifacts, etc.

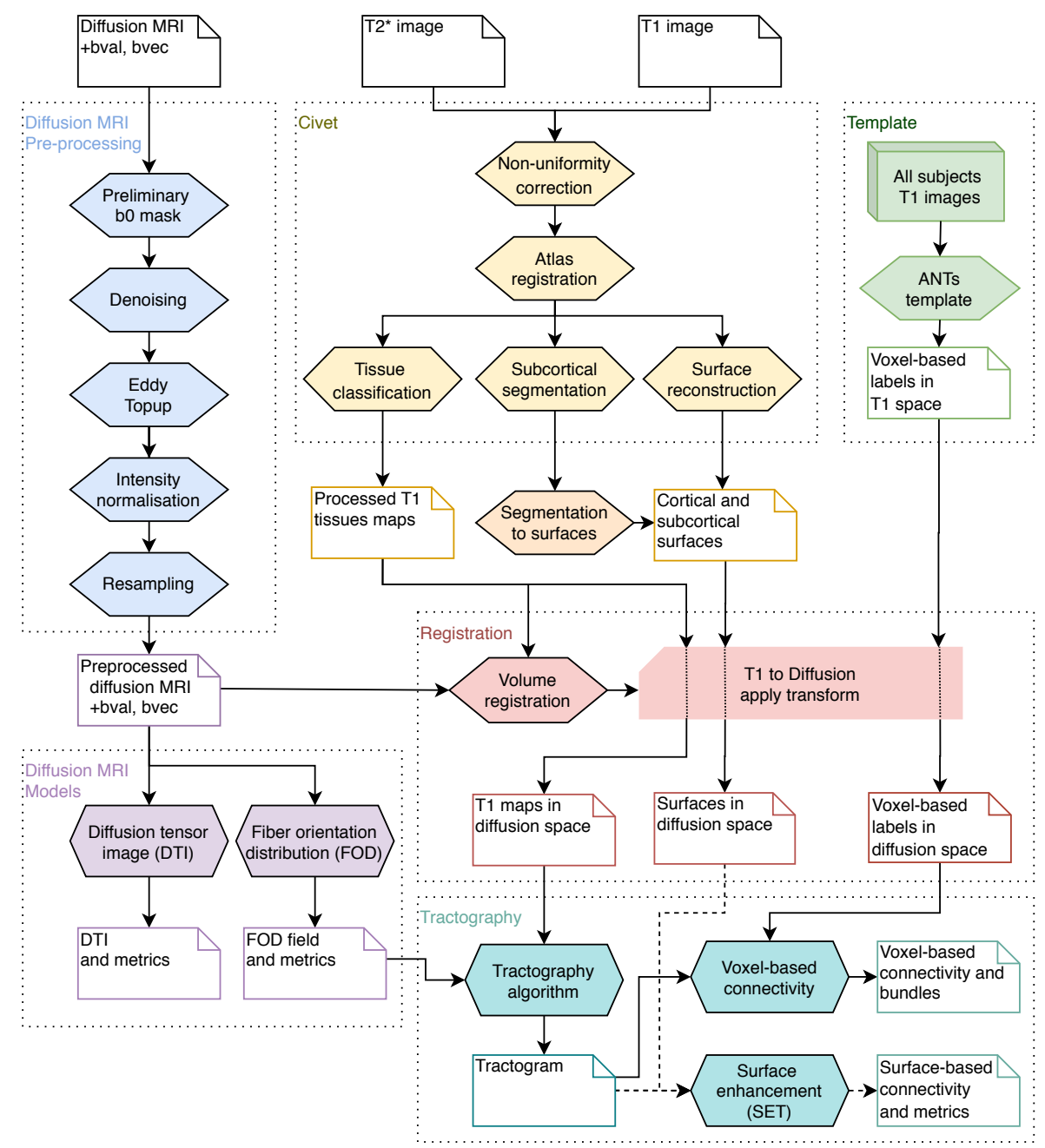

Figure 1: Processing pipeline architecture, for diffusion, $\mathrm{T} 1$ and $\mathrm{T} 2{ }^{\star}$ weighted images: dMRI pre-processing (blue), CIVET for WM-GM-CSF tissue maps with surfaces (yellow), T1 template reconstruction (green), Registration to align T1 maps from anatomical to diffusion space (red), diffusion local models reconstruction (purple), and tractography with structural connectivity (cyan). 


\section{Data conversion: DICOM to NIfTI}

Since most dMRI processing tools cannot directly input DICOM files, a conversion is necessary. First, DICOM ('.dcm') files were converted to Neuroimaging Informatics Technology Initiative (NIfTI) file format ('.nii') using dcm2niix [Li et al., 2016]. For dMRI volumes, this conversion also generates two accompa105 nying 'bval' and 'bvec' files which contain acquisition details of the b-values and b-vectors for each volume.

\section{Transformation (space) convention}

To facilitate the use of common dMRI software, all NIfTI files were modified to the RAS (left-to-right, posterior-to-anterior, inferior-to-superior) convention.

\section{Diffusion-weighted images (dMRI)}

Each dMRI acquisition is composed of a total of 32 diffusion-weighted threedimensional volumes: 30 with diffusion directions and 2 baseline (b0 images). Of the two b0 images, one is acquired in the anterior-to-posterior (A-P) direction, which is in alignment with all volumes of the main dMRI (named 'b0.nii'). The second b0 is acquired in the reverse direction, posterior-to-anterior $(\mathrm{P}-\mathrm{A})$, (named 'b0_rev.nii'). This reverse b0 is used to correct for susceptibility-induced distortion in dMRI. Each direction measures the signal loss along the chosen orientation (b-vector) with a single b-value of 1000 .

\subsection{Diffusion MRI Pre-processing}

A preliminary b0 mask ('bet_prelim_b0.nii') is estimated with FSL-bet from the 'b0.nii' dilated with MRtrix-mask filter. This is done to mask voxels determined to be outside of the brain tissue and to remove unnecessary computation of invalid voxels. Denoising was performed for all dMRI directions ('dwi.nii') inside the preliminary mask ('bet_prelim_b0.nii') with MRtrix-dMRIdenoise[Tournier et al., 2019].Topup correction map was computed with FSL-topup, to correct for common dMRI acquisition deformation, using both oppositely-acquired b0 images ('b0.nii', 'b0_rev.nii') and applied to all dMRI directions ('dwi.nii'). Eddy current correction was also applied using FSL-eddy. FSL-eddy applies both eddy current and distortion correction (generated from FSL-topup) to all diffusion volumes; it also returns distortion-corrected 'bval' and 'bvec' files. A new brain mask ('bet_b0.nii') is computed after the deformation correction, minimally dilated with MRtrix-mask filter. Intensity normalization was employed to establish spatial uniformity of the signal intensity across the dMRI volume using ANTs-N4BiasFieldCorrection. Additionally, MRtrix-dMRInormalise was used to harmonize the diffusion signal across subjects [Tournier et al., 2019]. Each diffusion volume was resampled with linear interpolation to an isotropic resolution of $1 \mathrm{~mm}$ [Lehmann et al., 1999; Dyrby et al., 2014].

\subsection{Diffusion MRI models}

DTI. Dipy-TensorModel was used to estimate the Diffusion Tensor Image (DTI) [Basser et al., 1994] at every spatial position (voxel), with a weighted 
least squares method. Subsequently, multiple DTI measures were computed from reconstructed tensors (Figure 2), including the Fractional Anisotropy (FA), Mean Diffusivity (MD), Apparent Diffusion Coefficient (ADC), Radial Diffusivity (RD), and red-green-blue (RGB) map colored in the $\mathrm{x}-\mathrm{y}-\mathrm{z}$ orientation (Figure 3).

FOD. Dipy-csdeconv package was used to compute fiber orientation distribution (FOD). Initially the "single fiber distribution" is estimated with the auto_response function and afterward the full distribution is computed at each position using ConstrainedSphericalDeconvModel with the symmetric724 discretized sphere. The FOD represents the estimated orientation distribution of fibrous structure at each voxel [Tournier et al., 2007; Descoteaux et al., 2009]. Peaks, representing main diffusion directions, are extracted from local maxima of each FOD angular distribution, similar to a vector field (Figure 2). This FOD field is later used to compute the tractography and estimate the structural 155 connectivity. In addition, some feature maps are estimated from the FOD reconstruction, illustrated in Figure 3, such as total apparent fiber density (AFD).

\subsection{T1w image processing}

Segmentation maps for white matter, gray matter, cerebrospinal fluid (CSF) and subcortical structures (SC) were generated by CIVET 2.1, from the native ${ }_{160} \mathrm{~T} 1$ anatomical image. A brain mask, also estimated from this segmentation, was applied to all T1w images before registration [Zijdenbos et al., 1998; Tohka et al., 2004]. Cortical surfaces were reconstructed with CIVET 2.1 [Kim et al., 2005; Lyttelton et al., 2007], similarly to FreeSurfer. Subcortical regions were estimated with ANIMAL [Collins et al., 1999] and converted into meshes using a marching cube algorithm, and then combined with cortical surfaces in a single file. The anatomical T1 and diffusion volume were co-registered, transforming the $\mathrm{T} 1$ volume into native diffusion space. The resulting transformations (affine matrix and warp) from antsRegistration, are also used to align subsequent anatomical maps and cortical surfaces to diffusion space [Avants et al., 170 2011; Theaud et al., 2020].

\section{Template construction}

In parallel to the included T1w processing within Tractoflow, a PING specific $\mathrm{T} 1 \mathrm{w}$ template was generated using antsMultivariateTemplateConstruction2.sh [Avants et al., 2011], which automates an iterative registration process to move 175 any number of $\mathrm{T} 1 \mathrm{w}$ volumes into a common space. Once the template has been generated, the resulting transformation files are used to register other templatealigned volumes into individual subject space. This is applied in subsequent processes which require aligning atlas segmentation labels into native $\mathrm{T} 1 \mathrm{w}$ and diffusion volume space, as described below in creating voxel-based connectivity matrices. Averaged tissue maps in the template space can be oberved in Figure 4. 
bioRxiv preprint doi: https://doi.org/10.1101/2020.11.24.396549; this version posted November 25, 2020. The copyright holder for this preprint (which was not certified by peer review) is the author/funder, who has granted bioRxiv a license to display the preprint in perpetuity. It is made available under aCC-BY-ND 4.0 International license.

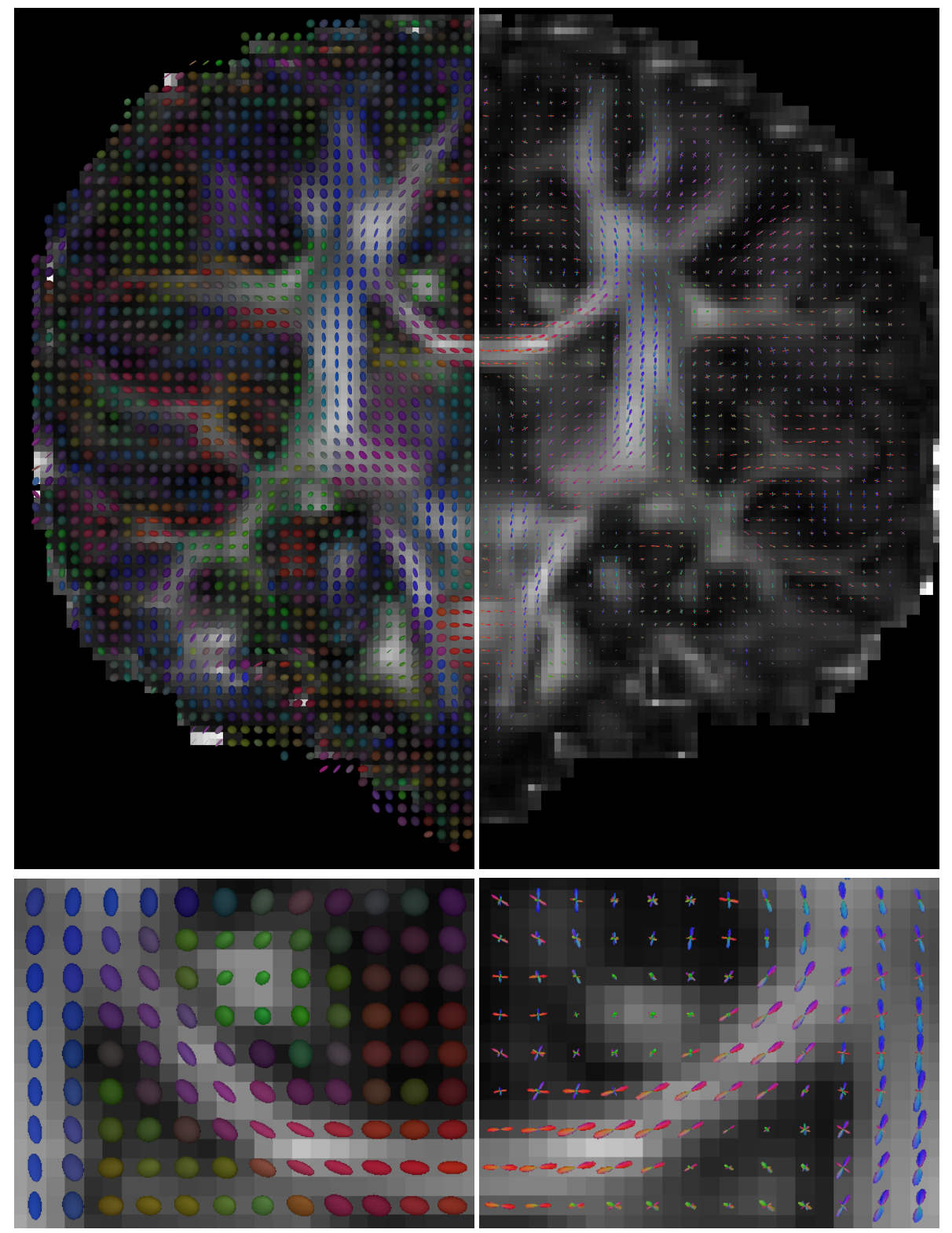

Figure 2: Coronal view of the reconstructed diffusion tensor image (DTI) on the left, and fiber orientation distribution (FOD) on the right. On the bottom row, a zoomed view of the Corpus Callosum and Cingulum regions. 
FA

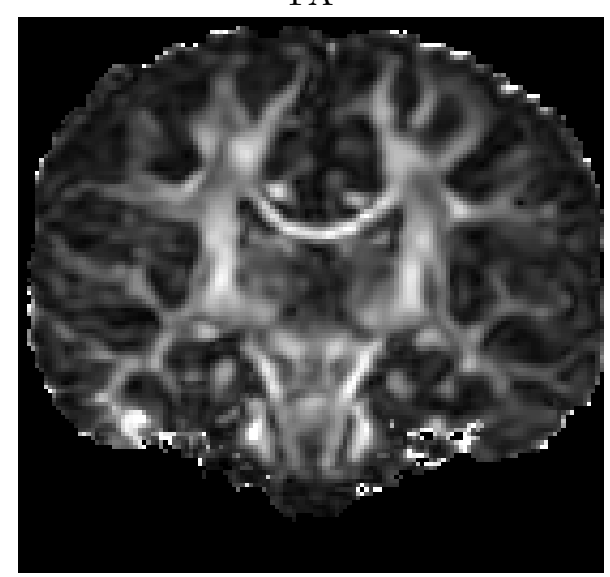

RGB

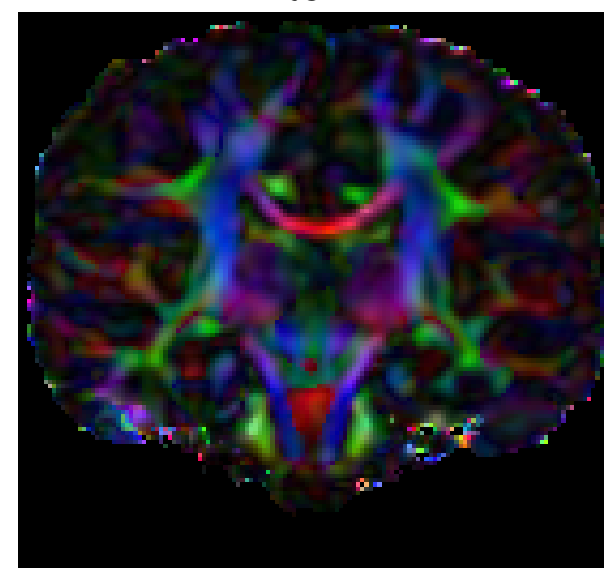

MD

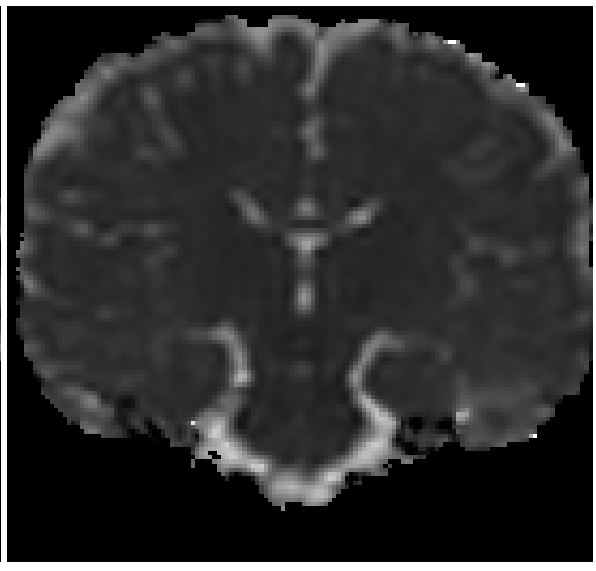

Total AFD

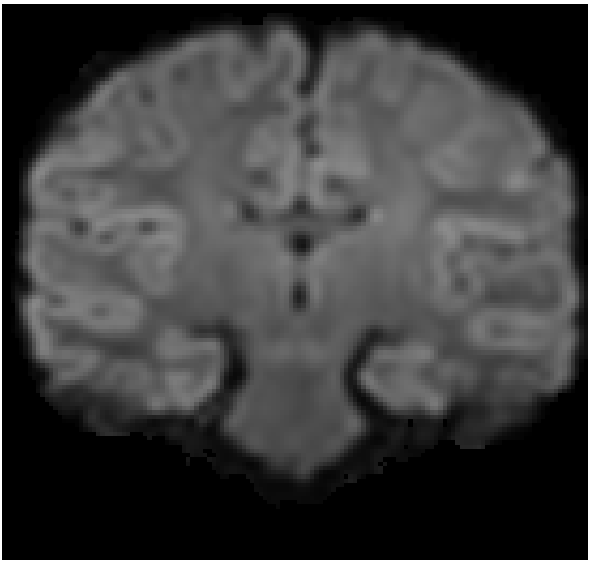

Figure 3: Diffusion tensor image (DTI) field measures: fractional anisotropy (FA), mean diffusivity (MD), and red-green-blue (RGB) map colored from the $x-y-z$ orientation. Total apparent fiber density (AFD) estimated from the Fiber orientation distribution (FOD).

\subsection{Structural connectivity analysis}

\section{Tractography}

Structural pathways can be reconstructed from dMRI by following the local orientation of estimated local models with a process called tractography. In this work, we used the "particle filtering tractography" (PFT) [Girard et al., 2014], implemented in Dipy, taking advantage of the sharp orientation from the FOD field. Resulting streamlines represent estimated pathways of the brain macrostructure and connectivity. Similarly to anatomically-constrained tractog190 raphy (ACT) [Smith et al., 2012], PFT takes advantage of previously computed WM-GM-CSF tissue maps, to define legal and illegal regions. 
The "surface-enhanced tractography" (SET) [St-Onge et al., 2018], an adaptation of the PFT algorithm, was also used to initialize and terminate streamlines from cortical and subcortical surfaces at the WM-GM boundary. Since CIVET 2.1 produces a standardized mesh, with a fixed number of vertices and triangles for every subject, SET allows for better comparison and individually variable correlation of connectivity features.

\section{Connectivity matrices}

Connectivity matrices were generated using two separate methods: voxeland surface-based. Voxel-based matrices were created using a 3D anatomical Desikan-Killiany-Tourville (DKT) [Desikan et al., 2006; Klein and Tourville, 2012] segmentation label in each acquisition's native diffusion space. To register the DKT segmentation label to native diffusion space, a number of image deformation steps were executed. The DKT segmentation label was moved from MNI space to the PING template space and then inversely warped to

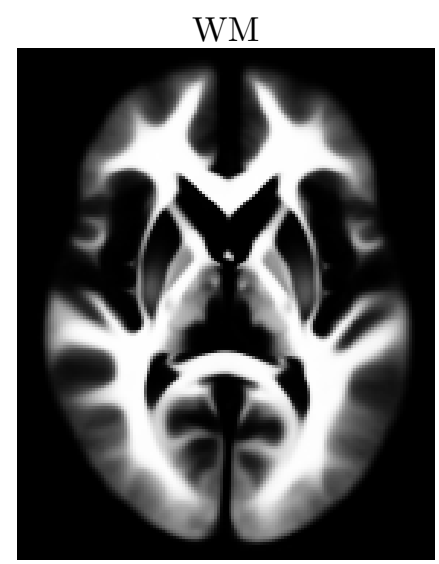

CSF

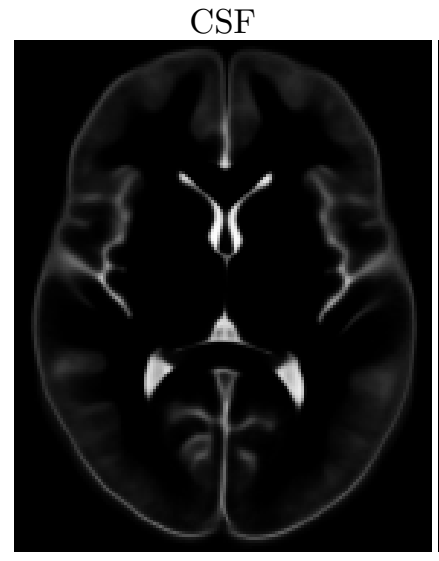

Figure 4: Averaged tissue maps in the template space, axial view.

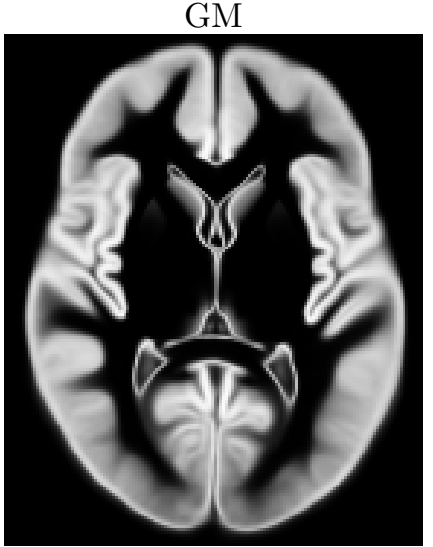

$\mathrm{SC}$

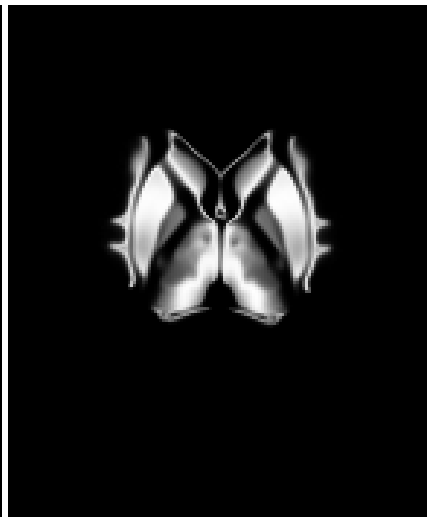


subject-specific $\mathrm{T} 1 \mathrm{w}$ native space. Using the transformation affine matrix and warp image from Tractoflow processing, the DKT label was then aligned from $\mathrm{T} 1 \mathrm{w}$ to native diffusion space. This native-space label was generated for each diffusion acquisition and used as input with the corresponding tractogram in 210 SCIL-scil_compute_robust_connectivity_matrix.py, which outputs a connectivity matrix as a npy file. Depicted in Figure 5, connectivity is represented as the number of streamlines with endpoints within a region of interest (ROI) label as delineated by the DKT label.

Using the surface-based method, every streamline generated by SET starts 215 and terminates within a triangle that makes up the cortical surface mesh. Each triangular facet corresponds to a specific cortical label in the CIVET DKT atlas. Subsequently, the connectivity matrix is computed by counting the number of streamlines connecting two different cortical regions.

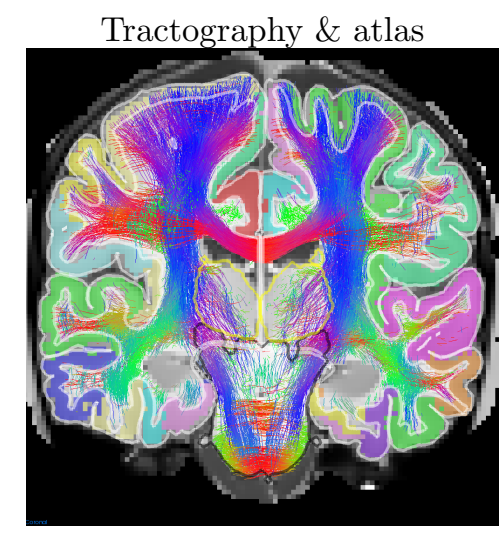

Connectivity 3D visualization

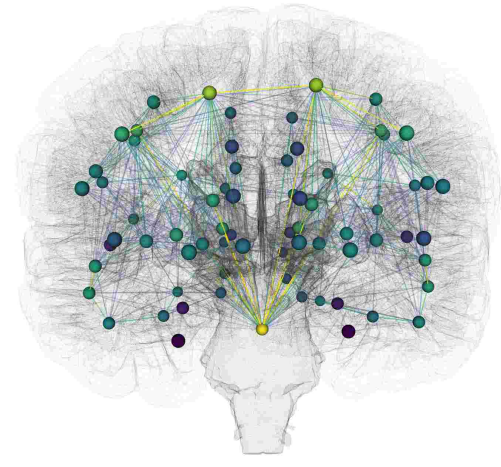

Voxel connectivity matrix

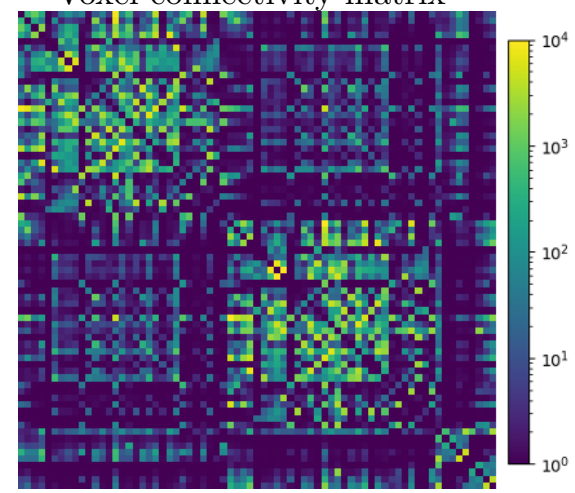

SET connectivity matrix

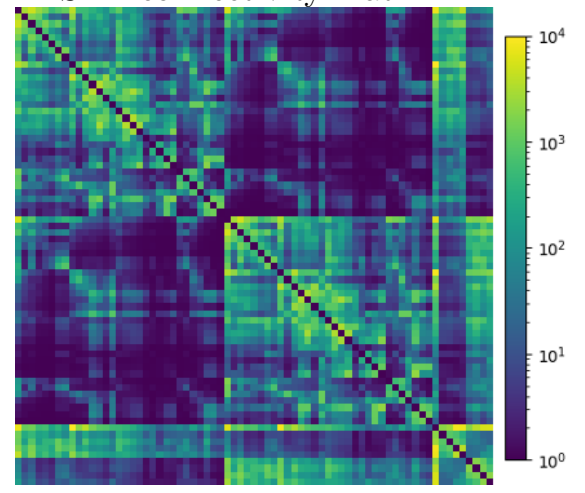

Figure 5: Tractography reconstruction visualized over the DKT atlas labels and resulting connectivity matrices, streamlines count per million in log scale. The connectivity matrix contains 35 cortical and subcortical labels per hemisphere (left, right) and the brainstem, for a total of $71 \times 71$ potential connections. 


\section{Technical Validation}

220

235

\section{DTI \& FOD}

Diffusion orientations acquired from the b-vector files and their respective eddy- and topup-corrected versions were validated by visualizing DTI main orientation in well-known brain structures: the corpus callosum and the cingulum. A flip in the $\mathrm{x}$ axis for b-vector values was required as the images were ac-

255 quired in LAS orientation, but Dipy is based on RAS reference. Resulting local reconstructions were also qualitatively verified for shape, size and orientation discrepancies (Figure 2). This ensured that computed DTI and FOD values were correct and well-aligned with the white matter structure. 


\begin{abstract}
Diffusion measures
260

Diffusion measures were also qualitatively validated using maps for FA, MD, RD, RGB and AFD. The variability of these dMRI metrics for this dataset is presented in the next sub-section. Subjects were removed if their diffusion maps presented visible artifacts such as motion, poor registration, uncorrected deformations or signal loss.
\end{abstract}

\title{
Tractograms
}

The resulting tractograms, from 100 randomly chosen subjects, were inspected with $3 \mathrm{D}$ visualization, to ensure no reconstruction artifacts were present.

\subsection{Dataset Variability}

In addition to standard quality control and visual validation, quantitative measures and variability were computed after each of the following processing steps. This was done to ensure that no major outliers remained in the final dataset. These variability results could also serve as references for future brain development studies or comparison with the PING dataset.

\section{Template alignment}

After the template reconstruction (Figure 4), an alignment score (dice) to this averaged template was computed for each individual probabilistic tissue map (Figure 6a). This template alignment score (mean; standard deviation) for WM (0.897; 0.010), GM (0.877; 0.015) and SC $(0.930 ; 0.019)$ is excellent, with small variance. The CSF score $(0.574 ; 0.039)$ is lower, compared to others, mostly due to brain mask variation. Subjects misaligned to the template were easily detected as outliers from the WM dice score (z-score $<-5)$ and confirmed through visual validation. In general, a data point is considered an outlier when the z-score is greater than 3 or less than -3. Removing these non-aligned subjects consistently reduced the dice standard deviation by a factor of 3 for WM 285 (around 2 for other tissue maps). Almost identical results and corresponding outliers were found using other distance measures (L1 and L2) as well.

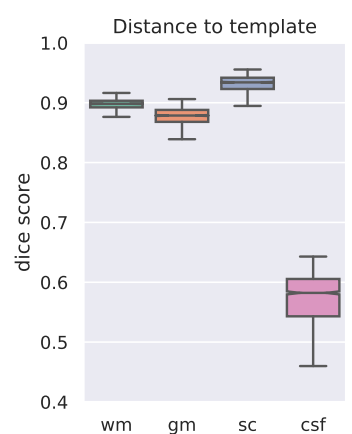

a)

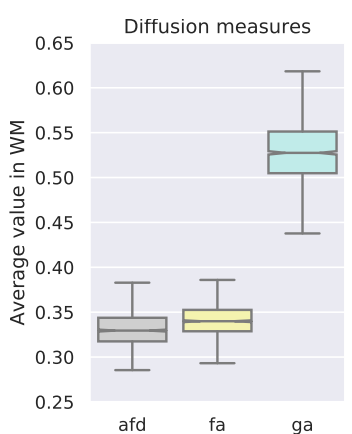

b)

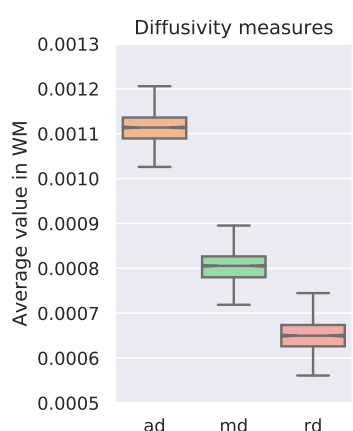

c)

Figure 6: a) Dice alignment score to the template, for each subject. White matter measures of: b) diffusion (AFD, FA, GA) and c) diffusivity in $\mathrm{mm}^{2} / \mathrm{s}$ (AD, MD, RD). 


\section{Diffusion measures}

Measures estimated from dMRI models (DTI and FOD) were also evaluated for each subject. Intensities in each tissue structure were analysed with a histogram and average value per subject (see Figure 6, Table A.1). From this variability analysis, results suggest that some FOD metrics, such as NUFO, are too variable to measure any group differences in this dataset. Nonetheless, this limitation could be partially caused by the variable nature of this dataset (age variation, multiple acquisition site, etc).

FOD model data response (frf)

Fiber response function (frf) was first estimated for each dMRI acquisition in the center of the WM structure in high FA values near the corpus callosum. Results were then compared for each scanner and acquisition site (Figure 7a, Table A.2). Since all frf were similar across PING, values used to compute FOD were fixed to $18,4,4$ (ratio of $18 / 4$ ), the dataset average.

\section{Connectivity matrices}

Connectivity matrices were employed to measure inter-subject distance. For subjects with multiple dMRI acquisitions, intra-subject distance was also measured. In addition, variability analysis was performed over connectivity matrices to estimate outliers and the differences between acquisition sites and age groups. Figure 7b displays L1-distance, from the average connectivity to each subject's connectivity matrix, grouped per acquisition site (Table A.3).

\section{Metrics}

Dice score and distance equations, in between images $A$ and $B$ at each voxel position $v$ in the whole $3 \mathrm{D}$ volume $\mathcal{V}$ is as follows:

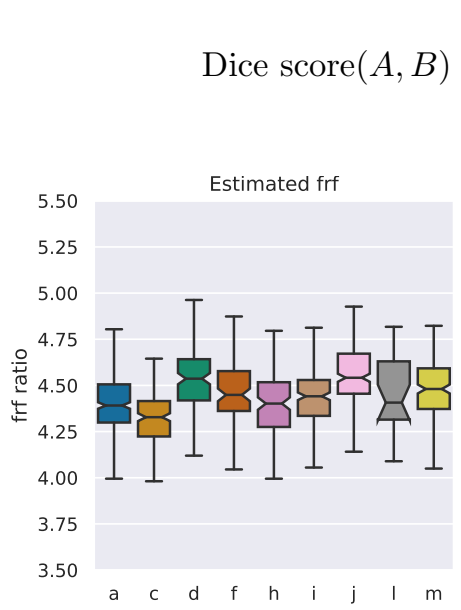

a)

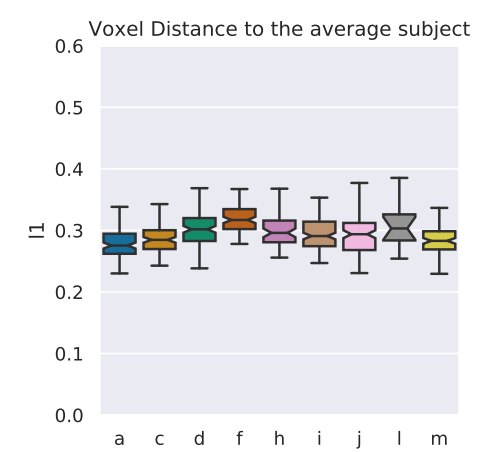

b)

Figure 7: a) Estimated fiber response function (frf) ratio, b) connectivity matrices distance to the average subject, across acquisition sites. MRI scanner for each site: Philips Achieva (a,h), GE Signa HDx (c), GE Discovery MR750 (i) and Siemens TrioTim (d,f,j,l,m). 


\section{Conclusion}

310

320

\section{Data Records}

Data and research tools used in the preparation of this manuscript were obtained from the National Institute of Mental Health (NIMH) Data Archive (NDA). NDA is a collaborative informatics system created by the National Insti345 tutes of Health to provide a national resource to support and accelerate research in mental health. Dataset identifier(s): 10.15154/1519178. This manuscript reflects the views of the authors and may not reflect the opinions or views of the $\mathrm{NIH}$ or of the Submitters submitting original data to NDA. Information on the 
original PING dataset, including acquisition protocols, is detailed in Jernigan

350

355 visualized and overlaid onto the T1 image with MI-Brain (see Figure 5).

DTI

Tensor images were saved with Dipy and Nibabel in NIfTI format. The resulting image is a $4 \mathrm{D}$ volume: spatially represented in $3 \mathrm{D}$ with a fourth dimension containing all 6 symmetric tensor coefficients. Tensor coefficients were saved in this given order along the fourth dimension axis, where: $\left[D_{00}, D_{01}\right.$, $\left.D_{02}, D_{11}, D_{12}, D_{22}\right]$, where

$$
D=\left[\begin{array}{lll}
D_{00} & D_{01} & D_{02} \\
D_{01} & D_{11} & D_{12} \\
D_{02} & D_{12} & D_{22}
\end{array}\right]
$$

FOD

375 $\quad$ FOD fields were saved with Dipy using the default spherical harmonics (SH) representation [Descoteaux et al., 2009]. Only even-ordered SH coefficients $\left(c_{l}^{m}\right)$ were saved, as computed FOD are symmetric. The SH file is ordered by $\mathrm{SH}$ order (l) first and phase $(m)$ second: $\left[c_{0}^{0} ; c_{2}^{-2}, c_{2}^{-1}, c_{2}^{0}, c_{2}^{1}, c_{2}^{2} ; c_{4}^{-4}, c_{4}^{-3}, c_{4}^{-2}, c_{4}^{-1}, c_{4}^{0}, c_{4}^{1}, c_{4}^{2}\right.$, $\left.c_{4}^{3}, c_{4}^{4} ; c_{6}^{-6}, c_{6}^{-5}, c_{6}^{-4}, c_{6}^{-3}, c_{6}^{-2}, c_{6}^{-1}, c_{6}^{0}, c_{6}^{1}, c_{6}^{2}, c_{6}^{3}, c_{6}^{4}, c_{6}^{5}, c_{6}^{6}\right]$. 
395

\section{Tractograms}

Tractography reconstructions were saved in TrackVis format ('.trk'). This format is well supported in Nibabel, Dipy and MI-Brain.

Connectivity matrices

All resulting connectivity matrices were stored as nummpy array matrices ('.npy') format. The ordered list of labels is given in the 'labels_co_matrix.txt' file.

\section{Usage Notes}

\section{Command lines}

Nextflow and Singularity need to be installed; a complete example is given in 'PING_example.sh'.

nextflow run civet-nf/main.nf --civet path/to/civet'output/ " -with-singularity set ping.img

sh civet-nf/tree'for tractoflow.sh -f path/to/data/ -c civet-nf/results/ " -o tractoflow input/

nextflow run tractoflow-pve/main.nf --root tractoflow input/ " --dti shells "0 1000" --fodf shells "0 1000" "

-profile civet"pve -with-singularity tractoflow'2.0.0.img

nextflow run set-nf/main.nf --tractoflow tractoflow inf/results/ " --civet path/to/civet output/ -profile civet DKT “

-with-singularity set'ping.img

\section{Pediatric Imaging Neurocognition and Genetics (PING)}

Data collection and subsequent dataset for this project were obtained from the Pediatric Imaging, Neurocognition and Genetics Study (PING), National Institutes of Health Grant RC2DA029475. PING is funded by the National Institute on Drug Abuse and the Eunice Kennedy Shriver National Institute of Child Health \& Human Development. PING data are disseminated by the PING Coordinating Center at the Center for Human Development, University of Cal410 ifornia, San Diego. PING data (nda.nih.gov/edit_collection.html?id=2607) and Consortium (ping-dataportal.ucsd.edu/sharing/Authors10222012.pdf), detailed in Jernigan et al. [2016].

\section{Acknowledgements}

Supported by grants from Brain Canada (238990, 243030), CFREF/HBHL 415 Innovative Ideas (247613), Coutu Research Fund (241177), CFREF/HBHL Discovery (247712), Université de Sherbrooke Research Chair in NeuroInformatics and NSERC Discovery Grant. 


\section{Author contributions statement}

The concept of this work, processing the structural connectivity of the PING dataset, was proposed and realized by Noor B Al-Sharif, supervised by Alan C Evans. Diffusion MRI processing planning and work outline was done by Etienne St-Onge, supervised by Maxime Descoteaux. Tractoflow pipeline initial implementation, and technical assistance was done by Guillaume Theaud. Processing pipeline adaptation for CIVET tissue maps, surfaces and SET was implemented by Etienne St-Onge, and rigorously tested by Noor B Al-Sharif, resulting in Tractoflow-pve.

\section{Conflict of Interest}

Maxime Descoteaux is co-founder of Imeka Solutions Inc. Other authors declare they have no actual or potential competing financial interests.

\section{Data Citations}

Data and research tools used in the preparation of this manuscript were obtained from the National Institute of Mental Health (NIMH) Data Archive (NDA). NDA is a collaborative informatics system created by the National Institutes of Health to provide a national resource to support and accelerate research 435 in mental health. Dataset identifier(s): 10.15154/1519178. This manuscript reflects the views of the authors and may not reflect the opinions or views of the NIH or of the Submitters submitting original data to NDA.

\section{References}

Avants, B.B., Tustison, N.J., Song, G., Cook, P.A., Klein, A., Gee, J.C., 2011. A 440 reproducible evaluation of ants similarity metric performance in brain image registration. Neuroimage 54, 2033-2044.

Basser, P.J., Mattiello, J., LeBihan, D., 1994. Mr diffusion tensor spectroscopy and imaging. Biophysical journal 66, 259-267.

Benes, F.M., Turtle, M., Khan, Y., Farol, P., 1994. Myelination of a key relay zone in the hippocampal formation occurs in the human brain during childhood, adolescence, and adulthood. Archives of general psychiatry 51, 477-484.

Collins, D.L., Zijdenbos, A.P., Baaré, W.F., Evans, A.C., 1999. Animal+ insect: improved cortical structure segmentation, in: Biennial International Conference on Information Processing in Medical Imaging, Springer. pp. 210-223.

Dell'Acqua, F., Tournier, J.D., 2019. Modelling white matter with spherical deconvolution: How and why? NMR in Biomedicine 32, e3945. 
Deoni, S.C., Mercure, E., Blasi, A., Gasston, D., Thomson, A., Johnson, M., Williams, S.C., Murphy, D.G., 2011. Mapping infant brain myelination with magnetic resonance imaging. Journal of Neuroscience 31, 784-791.

Descoteaux, M., 2015. High angular resolution diffusion imaging (hardi). Wiley Encyclopedia of Electrical and Electronics Engineering , 1-25.

Descoteaux, M., Deriche, R., Knösche, T.R., Anwander, A., 2009. Deterministic and probabilistic tractography based on complex fibre orientation distributions. Medical Imaging, IEEE Transactions on 28, 269-286.

Desikan, R.S., Ségonne, F., Fischl, B., Quinn, B.T., Dickerson, B.C., Blacker, D., Buckner, R.L., Dale, A.M., Maguire, R.P., Hyman, B.T., et al., 2006. An automated labeling system for subdividing the human cerebral cortex on mri scans into gyral based regions of interest. Neuroimage 31, 968-980.

Dyrby, T.B., Lundell, H., Burke, M.W., Reislev, N.L., Paulson, O.B., Ptito, M., Siebner, H.R., 2014. Interpolation of diffusion weighted imaging datasets. NeuroImage 103, 202-213.

Giedd, J.N., Rapoport, J.L., 2010. Structural mri of pediatric brain development: what have we learned and where are we going? Neuron $67,728-734$.

470 Girard, G., Whittingstall, K., Deriche, R., Descoteaux, M., 2014. Towards quantitative connectivity analysis: reducing tractography biases. Neuroimage 98, 266-278.

Glasser, M.F., Sotiropoulos, S.N., Wilson, J.A., Coalson, T.S., Fischl, B., Andersson, J.L., Xu, J., Jbabdi, S., Webster, M., Polimeni, J.R., et al., 2013.

${ }_{475}$ The minimal preprocessing pipelines for the human connectome project. Neuroimage 80, 105-124.

Jbabdi, S., Sotiropoulos, S.N., Haber, S.N., Van Essen, D.C., Behrens, T.E., 2015. Measuring macroscopic brain connections in vivo. Nature neuroscience $18,1546-1555$.

Jernigan, T.L., Baaré, W.F., Stiles, J., Madsen, K.S., 2011. Postnatal brain development: structural imaging of dynamic neurodevelopmental processes, in: Progress in brain research. Elsevier. volume 189, pp. 77-92.

Jernigan, T.L., Brown, T.T., Hagler Jr, D.J., Akshoomoff, N., Bartsch, H., Newman, E., Thompson, W.K., Bloss, C.S., Murray, S.S., Schork, N., et al., 2016. The pediatric imaging, neurocognition, and genetics (ping) data repository. Neuroimage 124, 1149-1154.

Jeurissen, B., Descoteaux, M., Mori, S., Leemans, A., 2019. Diffusion mri fiber tractography of the brain. NMR in Biomedicine 32, e3785.

Jones, D.K., 2008. Studying connections in the living human brain with diffusion mri. cortex 44, 936-952. 
Jones, D.K., Cercignani, M., 2010. Twenty-five pitfalls in the analysis of diffusion mri data. NMR in Biomedicine 23, 803-820.

Kim, J.S., Singh, V., Lee, J.K., Lerch, J., Ad-Dab'bagh, Y., MacDonald, D., Lee, J.M., Kim, S.I., Evans, A.C., 2005. Automated 3-d extraction and evaluation of the inner and outer cortical surfaces using a laplacian map and partial volume effect classification. Neuroimage 27, 210-221.

Klein, A., Tourville, J., 2012. 101 labeled brain images and a consistent human cortical labeling protocol. Frontiers in neuroscience 6, 171.

Klingberg, T., Vaidya, C.J., Gabrieli, J.D., Moseley, M.E., Hedehus, M., 1999. Myelination and organization of the frontal white matter in children: a diffusion tensor mri study. Neuroreport 10, 2817-2821.

Lebel, C., Walker, L., Leemans, A., Phillips, L., Beaulieu, C., 2008. Microstructural maturation of the human brain from childhood to adulthood. Neuroimage 40, 1044-1055.

Lehmann, T.M., Gonner, C., Spitzer, K., 1999. Survey: Interpolation methods in medical image processing. IEEE transactions on medical imaging 18, 10491075 .

Lenroot, R.K., Giedd, J.N., 2006. Brain development in children and adolescents: insights from anatomical magnetic resonance imaging. Neuroscience \& biobehavioral reviews 30, 718-729.

Li, X., Morgan, P.S., Ashburner, J., Smith, J., Rorden, C., 2016. The first step for neuroimaging data analysis: Dicom to nifti conversion. Journal of neuroscience methods 264, 47-56.

Lim, K.O., Helpern, J., 2002. Neuropsychiatric applications of dti-a review. NMR in Biomedicine: An International Journal Devoted to the Development and Application of Magnetic Resonance In Vivo 15, 587-593.

Lyttelton, O., Boucher, M., Robbins, S., Evans, A., 2007. An unbiased iterative group registration template for cortical surface analysis. Neuroimage 34, $1535-1544$.

Mukherjee, P., Miller, J.H., Shimony, J.S., Philip, J.V., Nehra, D., Snyder, A.Z., Conturo, T.E., Neil, J.J., McKinstry, R.C., 2002. Diffusion-tensor mr imaging of gray and white matter development during normal human brain maturation. American Journal of Neuroradiology 23, 1445-1456.

Nagy, Z., Westerberg, H., Klingberg, T., 2004. Maturation of white matter ${ }_{525}$ is associated with the development of cognitive functions during childhood. Journal of cognitive neuroscience 16, 1227-1233.

Olesen, P.J., Nagy, Z., Westerberg, H., Klingberg, T., 2003. Combined analysis of dti and fmri data reveals a joint maturation of white and grey matter in a fronto-parietal network. Cognitive Brain Research 18, 48-57. 
Paus, T., Zijdenbos, A., Worsley, K., Collins, D.L., Blumenthal, J., Giedd, J.N., Rapoport, J.L., Evans, A.C., 1999. Structural maturation of neural pathways in children and adolescents: in vivo study. Science 283, 1908-1911.

Prayer, D., Prayer, L., 2003. Diffusion-weighted magnetic resonance imaging of cerebral white matter development. European journal of radiology 45, $235-243$.

Qiu, A., Mori, S., Miller, M.I., 2015. Diffusion tensor imaging for understanding brain development in early life. Annual review of psychology 66, 853-876.

Shatz, C.J., 1990. Impulse activity and the patterning of connections during cns development. Neuron 5, 745-756.

${ }_{540}$ Smith, R.E., Tournier, J.D., Calamante, F., Connelly, A., 2012. Anatomicallyconstrained tractography: improved diffusion mri streamlines tractography through effective use of anatomical information. Neuroimage 62, 1924-1938.

Sotiropoulos, S.N., Zalesky, A., 2019. Building connectomes using diffusion mri: why, how and but. NMR in Biomedicine 32, e3752.

545 Sowell, E.R., Thompson, P.M., Toga, A.W., 2004. Mapping changes in the human cortex throughout the span of life. The Neuroscientist 10, 372-392.

St-Onge, E., Daducci, A., Girard, G., Descoteaux, M., 2018. Surface-enhanced tractography (set). Neuroimage 169, 524-539.

Stiles, J., Jernigan, T.L., 2010. The basics of brain development. Neuropsychol550 ogy review 20, 327-348.

Theaud, G., Houde, J.C., Boré, A., Rheault, F., Morency, F., Descoteaux, M., 2020. Tractoflow: A robust, efficient and reproducible diffusion mri pipeline leveraging nextflow \& singularity. NeuroImage , 116889.

Tohka, J., Zijdenbos, A., Evans, A., 2004. Fast and robust parameter estimation for statistical partial volume models in brain mri. Neuroimage 23, 84-97.

Tournier, J.D., Calamante, F., Connelly, A., 2007. Robust determination of the fibre orientation distribution in diffusion mri: non-negativity constrained super-resolved spherical deconvolution. NeuroImage 35, 1459-1472.

Tournier, J.D., Smith, R., Raffelt, D., Tabbara, R., Dhollander, T., Pietsch, M., Christiaens, D., Jeurissen, B., Yeh, C.H., Connelly, A., 2019. Mrtrix3: A fast, flexible and open software framework for medical image processing and visualisation. NeuroImage, 116137.

Zijdenbos, A., Forghani, R., Evans, A., 1998. Automatic quantification of ms lesions in $3 \mathrm{~d}$ mri brain data sets: validation of insect, in: International Conference on Medical Image Computing and Computer-Assisted Intervention, Springer. pp. 439-448. 


\section{Appendix A. Full Comparison}

\begin{tabular}{|l|c|c|c|c|}
\hline & WM & GM & SC & CSF \\
\hline AD & $0.00111,0.000033$ & $0.00116,0.000072$ & $0.00104,0.000033$ & $0.00160,0.000146$ \\
MD & $0.00080,0.000033$ & $0.00103,0.000059$ & $0.00082,0.000032$ & $0.00144,0.000125$ \\
RD & $0.00065,0.000035$ & $0.00096,0.000054$ & $0.00070,0.000033$ & $0.00135,0.000117$ \\
AFD & $0.332,0.022$ & $0.332,0.026$ & $0.360,0.027$ & $0.244,0.030$ \\
FA & $0.340,0.019$ & $0.129,0.011$ & $0.254,0.015$ & $0.114,0.020$ \\
GA & $0.529,0.038$ & $0.191,0.026$ & $0.375,0.024$ & $0.190,0.050$ \\
dice & $0.897,0.010$ & $0.877,0.015$ & $0.930,0.019$ & $0.574,0.039$ \\
\hline
\end{tabular}

Table A.1: Diffusivity measures (AD, MD, RD) in $\mathrm{mm}^{2} / \mathrm{s}$, diffusion measures (AFD, FA, GA) and tissue maps dice alignment score, average \& variance over all subjects, for each segmentation maps.

\begin{tabular}{|l|c|c|c|}
\hline & first eigenvalue & second eigenvalue & ratio \\
\hline $\mathrm{a}$ & $0.001709,0.000124$ & $0.000388,0.000026$ & $4.40,0.16$ \\
$\mathrm{c}$ & $0.001686,0.000076$ & $0.000391,0.000019$ & $4.32,0.14$ \\
d & $0.001887,0.000196$ & $0.000416,0.000041$ & $4.54,0.18$ \\
$\mathrm{f}$ & $0.001850,0.000207$ & $0.000413,0.000041$ & $4.47,0.17$ \\
$\mathrm{~h}$ & $0.001836,0.000201$ & $0.000418,0.000039$ & $4.39,0.18$ \\
$\mathrm{i}$ & $0.001716,0.000087$ & $0.000387,0.000019$ & $4.44,0.16$ \\
$\mathrm{j}$ & $0.001932,0.000211$ & $0.000425,0.000042$ & $4.54,0.18$ \\
$\mathrm{l}$ & $0.001735,0.000128$ & $0.000390,0.000022$ & $4.45,0.18$ \\
$\mathrm{~m}$ & $0.001782,0.000183$ & $0.000397,0.000036$ & $4.49,0.19$ \\
\hline
\end{tabular}

Table A.2: Estimated fiber response function (frf) in $\mathrm{mm}^{2} / \mathrm{s}$ across acquisition sites: first eigenvalue, second eigenvalue, ratio. Philips Achieva (a,h), GE Signa HDx (c), GE Discovery MR750 (i) and Siemens TrioTim (d,f,j,l,m).

\begin{tabular}{|l|c|c|c|c|}
\hline & voxel L1 & voxel L2 & SET L1 & SET L2 \\
\hline a & $0.278,0.027$ & $0.0234,0.0029$ & $0.385,0.040$ & $0.0331,0.0055$ \\
c & $0.287,0.026$ & $0.0242,0.0032$ & $0.382,0.036$ & $0.0303,0.0068$ \\
d & $0.301,0.028$ & $0.0254,0.0030$ & $0.375,0.041$ & $0.0278,0.0050$ \\
f & $0.317,0.024$ & $0.0266,0.0031$ & $0.417,0.041$ & $0.0331,0.0058$ \\
h & $0.299,0.026$ & $0.0251,0.0029$ & $0.435,0.037$ & $0.0370,0.0048$ \\
i & $0.291,0.024$ & $0.0245,0.0028$ & $0.358,0.038$ & $0.0269,0.0051$ \\
j & $0.291,0.033$ & $0.0243,0.0033$ & $0.378,0.044$ & $0.0292,0.0057$ \\
l & $0.310,0.031$ & $0.0252,0.0027$ & $0.429,0.038$ & $0.0347,0.0063$ \\
m & $0.283,0.023$ & $0.0236,0.0026$ & $0.363,0.039$ & $0.0274,0.0052$ \\
\hline
\end{tabular}

Table A.3: Connectivity matrices L1, L2 and $\chi^{2}$ distances, per acquisition site, to the average matrix. Philips Achieva (a,h), GE Signa HDx (c), GE Discovery MR750 (i) and Siemens $\operatorname{TrioTim}(\mathrm{d}, \mathrm{f}, \mathrm{j}, \mathrm{l}, \mathrm{m})$. 